\title{
Mini-review
}

\section{Single molecule force spectroscopy on ligand-DNA complexes: from molecular binding mechanisms to biosensor applications}

\author{
Robert Ros $^{\mathrm{a}, *}$, Rainer Eckel ${ }^{\mathrm{a}}$, Frank Bartels ${ }^{\mathrm{a}}$, Andy Sischka ${ }^{\mathrm{a}}$, Birgit Baumgarth ${ }^{\mathrm{b}}$, \\ Sven David Wilking ${ }^{c}$, Alfred Pühler ${ }^{\mathrm{b}}$, Norbert Sewald ${ }^{\mathrm{c}}$, \\ Anke Becker ${ }^{b}$, Dario Anselmetti ${ }^{a}$ \\ ${ }^{a}$ Experimental Biophysics, Faculty of Physics, Bielefeld University, Universitätsstrasse 25, 33615 Bielefeld, Germany \\ ${ }^{\mathrm{b}}$ Genetics, Faculty of Biology, Bielefeld University, Universitätsstrasse 25, 33615 Bielefeld, Germany \\ c Organic and Bioorganic Chemistry, Faculty of Chemistry, Bielefeld University, Universitätsstrasse 25, 33615 Bielefeld, Germany
}

Received 15 December 2003; received in revised form 8 April 2004; accepted 16 April 2004

\begin{abstract}
Recent developments in single molecule force spectroscopy (SMFS) allow direct observation and measurements of forces that hold protein-DNA complexes together. Furthermore, the mechanics of double-stranded (ds) DNA molecules in the presence of small binding ligands can be detected. The results elucidate molecular binding mechanisms and open the way for ultra sensitive and powerful biosensor applications.
\end{abstract}

Keywords: Single molecule force spectroscopy; Atomic force microscopy; Optical tweezers; Ligand-DNA interaction

\section{Introduction}

The interaction of ligands with double-stranded (ds) DNA is fundamental for many intracellular processes and therefore of biotechnological relevance. For example proteins that bind to specific DNA target sequences control processes of transcription, translation and regulation of genes. Small binding ligands with reduced or non sequence specificity are often able to interfere with those processes and thus are

\footnotetext{
* Corresponding author. Tel.: +49-521-1065388; fax: +49-521-1062959.

E-mail address: robert.ros@physik.uni-bielefeld.de (R. Ros).
}

commonly used as anti-cancer drugs and antibiotics. Recent developments of ultrasensitive force transducers (Bustamante et al., 2000a) allow the investigation and manipulation of these interactions at the single molecule level. The most prominent techniques are based on atomic force microscopy (AFM) (Binnig et al., 1986) and optical tweezers (OT) (Ashkin et al., 1986).

In contrast to the well-established ensemble measurements, experiments dealing with single molecules have the potential to detect fast intermediate transition states, details of the energy landscape, and structural changes, and identifying "individual" behavior. Force is a direct observable to describe the interac- 
tion between different molecules or compartments in a macromolecular assembly. In single molecule force spectroscopy (SMFS) experiments the force response is detected while a complex formed by two molecules is separated or a single chain-like molecule is stretched.

In this paper, we review SMFS experiments of the binding of sequence specific proteins to dsDNA and of small ligands which bind in a mainly sequence unspecific manner to dsDNA.

\section{Single molecule force spectroscopy}

In force spectroscopy experiments, the complex or molecule of interest is stretched with a sub nanometer precise piezoelectric stage, while an ultrasensitive transducer detects the restoring force. The experiments reported in this review are based on AFM and OT (Fig. 1). In AFM the bending of a micro-fabricated

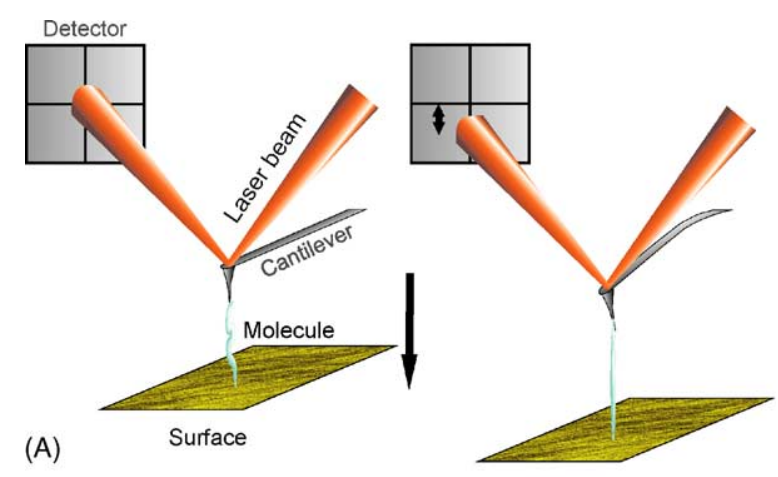

(B)

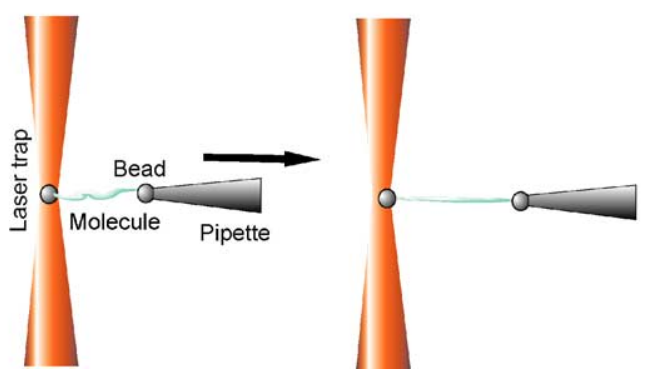

Fig. 1. Schematic force spectroscopy setup (A) based on AFM (B) based on OT technology. The molecule or molecule complex of interest is attached between the AFM tip and surface or the two beads. While increasing the distance between the points of attachments with sub nanometer accuracy, the restoring force is detected. cantilever is mostly detected by the deflection of a laser beam (Meyer and Amer, 1988). Spring constants of the cantilever in the order of $10-100 \mathrm{nN} / \mathrm{nm}$ and deflection sensitivities in the sub nanometer range allow measurements of forces from about $10 \mathrm{pN}$ to $10 \mu \mathrm{N}$. At the end of the cantilever, a tip with a radius of about $10 \mathrm{~nm}$ is integrated, which results in a unique spatial resolution in the nanometer range. In OT experiments a micrometer sized bead with a different refractive index is trapped in an electric field gradient of a laser focus by optical forces (Gordon, 1973; Ashkin, 1997). Displacements of the bead from the center of the laser focus can be converted into forces. Optical tweezers possess a superb force resolution in the sub piconewton range. However, because of the low spring constants of the optical trap $(<1 \mathrm{pN} / \mathrm{nm})$ the maximal force is smaller than $200 \mathrm{pN}$. Both inter- and intramolecular forces can be addressed by force spectroscopy experiments. To extract information about intermolecular forces, one binding partner is attached to the force transducer, i.e. the AFM tip or the OT bead, and the other to the translation stage. For successful ligand-receptor experiments a stable and directed immobilization is crucial (Ros et al., 1998). In a thermodynamically driven system the measured separation forces, depend on the loading rate that is exerted on the bound complex, i.e. the time dependency of the external forces (loading rate $=$ retract velocity $\times$ molecule elasticity). A slowly increasing load provides ample time for thermal fluctuations to drive the system over the energy barrier of the binding potential, resulting in small unbinding forces (Evans and Ritchie, 1997). Mapping the binding forces for different loading rates is often termed as dynamic force spectroscopy (DFS) and has been reviewed by Evans (2001). A new data analysis method for DFS experiments has been proposed (Evstigneev and Reimann, 2003) and was applied to specific protein-DNA data in this Special Issue.

Various ligand-receptor systems have been analyzed in this way on a single molecule level mainly with AFM technology. Pioneering work was done on biotin/avidin and biotin/streptavidin (Lee et al., 1993; Florin et al., 1994), complementary DNA strands (Lee et al., 1994; Strunz et al., 1999), selectins (Fritz et al., 1998) and antibody-antigen complexes (Dammer et al., 1996; Hinterdorfer et al., 1996; Schwesinger et al., 2000). Dynamic force spectroscopy exper- 
iments on complexes allow direct insights in the energy landscape by means of mapping activation energy barriers (Merkel et al., 1999). In addition, the biological function of the observed molecule can be addressed by the extraction of the thermal off-rates (Schwesinger et al., 2000). Intramolecular forces, on the other hand, have a direct influence on the mechanics of a molecule. Pathbreaking elasticity studies on single DNA molecules were shown with OT (Bustamante, 1994) and AFM (Rief et al., 1999). The latter describing the sequence dependence of the mechanics and extracts the base pair unzipping force for $\mathrm{G}-\mathrm{C}$ and $\mathrm{A}-\mathrm{T}$. Conformational changes in polysaccharides were observed and corroborated by molecular dynamic calculations (Rief et al., 1997b). The fundamental field of protein folding was addressed by unfolding individual domains of the giant muscle protein titin (Rief et al., 1997a) and the detection of intermediates (Marszalek et al., 1999). Here the combination with molecular dynamic simulations results in an atomistic view of structural chances in the unfolding process.

\section{Sequence specific binding of proteins to DNA}

The specific binding of proteins to a DNA target sequence is of fundamental interest for many biological questions. Recently, this interaction was investigated by two different SMFS techniques.

\subsection{Unzipping force analysis of protein association}

Koch et al. (2002) investigated the binding of the restriction enzymes BsoBI, XhoI, and EcoRI with a new technique based on unzipping the two strands of dsDNA with OT (Fig. 2). The tension force and extension of the DNA were recorded continuously. A bound protein is indicated by an increase in the force. This method allows the containment of the binding site on the DNA to about $25 \mathrm{bp}$, whereas repetition of the unzipping experiments lead to predictions about equilibrium constants. Life times from $92 \mathrm{~s}$ for $B s o \mathrm{BI}$ to $6000 \mathrm{~s}$ for $\mathrm{XhoI}$ and information about the energy landscape were extracted by DFS measurements with loading rates from 12 to $1200 \mathrm{pN} / \mathrm{s}$ (Koch and Wang, 2003).
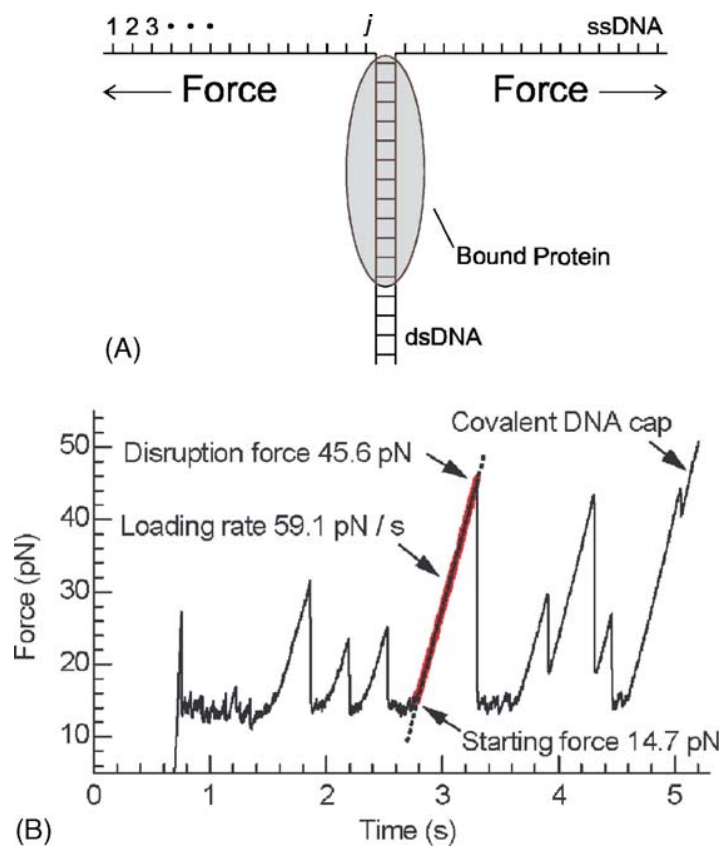

Fig. 2. Specific enzyme-DNA interaction analyzed by unzipping DNA. (A) Schematic setup and (B) typical force vs. time graph acquired at a constant loading rate. (Reprinted figure with permission from S.J. Koch, M.D. Wang, Phys. Rev. Lett. 91 (2003). Copyright (2004) by American Physical Society.)

\subsection{Direct force spectroscopy on protein-DNA complexes}

Direct force measurements by mechanically unbinding protein-DNA complexes were reported by Bartels et al. for a transcriptional regulator protein that binds to three promotor regions in a gene cluster (Bartels et al., 2003) (see Fig. 3). Three DNA fragments comprising the target sequences were selected for the force spectroscopy measurements and attached covalently via a long flexible polymer linker to the AFM tip while the protein was anchored covalently on the surface. DFS experiments with loading rates from $70 \mathrm{pN} / \mathrm{s}$ to $79 \mathrm{nN} / \mathrm{s}$ allow discrimination of the complex lifetimes and the detection of inner barriers in the energy landscape. From the lower loading rate regime, a thermal off-rate of $k_{\text {off }}=(1.2 \pm 1.0) \times 10^{-3} \mathrm{~s}^{-1}$ corresponding to a life time of about $830 \mathrm{~s}$ was derived for all promotor regions. In the higher loading rate regime distinct differences, that were attributed to the molecular binding mechanism, were observed. 

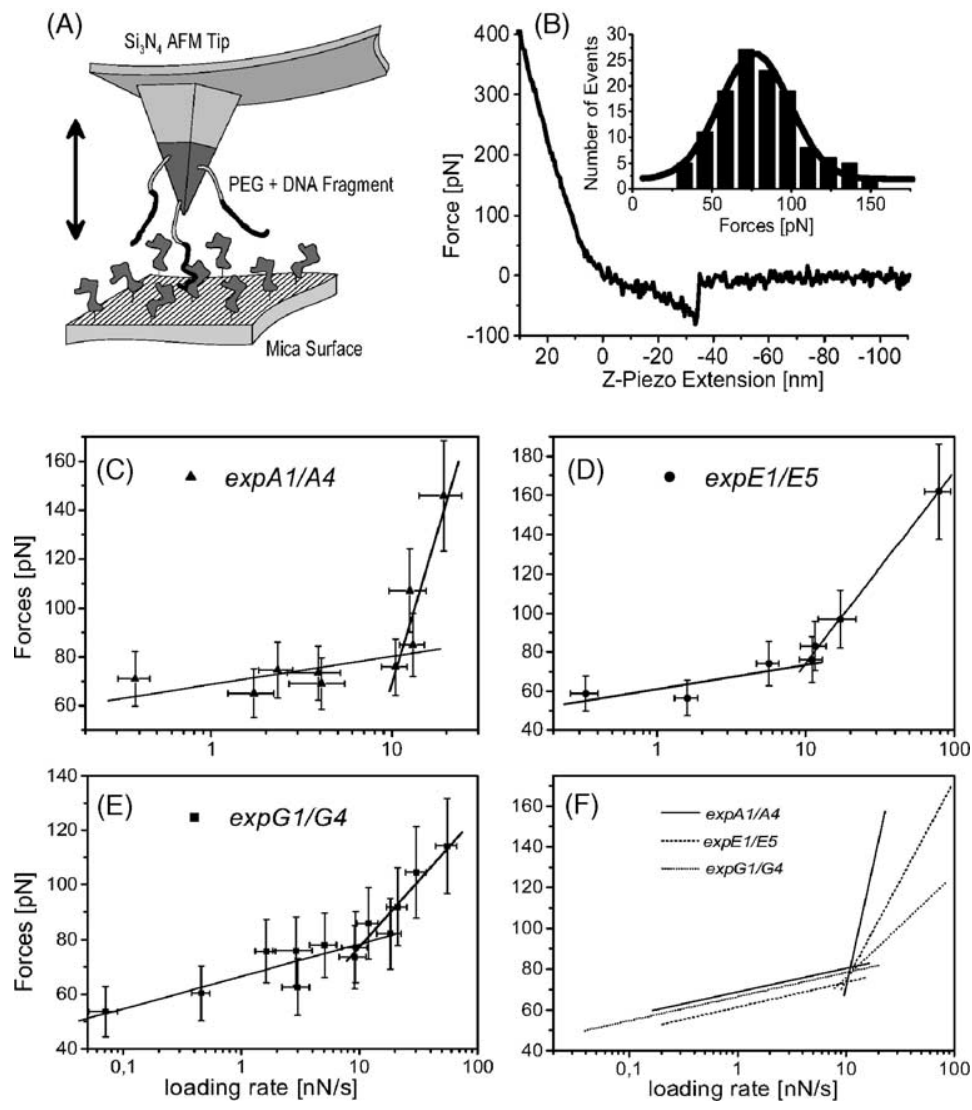

Fig. 3. Specific regulatory protein-DNA interaction analyzed with AFM based SMFS (A) schematic setup (B) typical force distance curve (only the retracting part) with a rupture event. The rupture forces of a given series are combined to form a histogram with a gaussian like distribution (inset). (C-E) Loading rate dependent force measurements of three different DNA fragments with a regulatory protein. (F) Comparison of the fits from (C) to (E). Different slopes correspond to inner and outer barriers. (Reprinted from publication (Bartels, 2003), with permission from Elsevier.)

\section{Interaction of small ligands with DNA}

The mechanical properties of single dsDNA were investigated in details in the pioneering works of Bustamante (1994) with OT and Rief et al. (1999) with AFM. Briefly (see Fig. 4b), the DNA molecule in solution behaves like an entropic coil. Pulling on the two ends induces a restoring force due to decreasing degrees of freedom. This effect dominates the stretching behavior up to about $50-60 \mathrm{pN}$ and can be described in good agreement with the worm-like chain (WLC) elasticity model (Bustamante et al., 2000b). The extension at this point can be interpreted as the contour length of the dsDNA. At higher forces a distinct plateau is visible, corresponding to a struc- tural transition from B-DNA to a stretched form, the so-called S-DNA. In this cooperative transition the molecule can be elongated by a factor of $0.6-0.8$ by an only weak increase in the restoring force (Smith et al., 1996; Cluzel et al., 1996).

The force value of this transition depends on the dsDNA sequence (Rief et al., 1999) and experimental conditions (Wenner et al., 2002). Higher forces induce the melting of the two complementary DNA strands followed by single strand stretching and the detachment of the molecule from the force transducer or translation stage. Fig. 4 shows the mechanics of a single poly(dG-dC) dsDNA molecule explored with AFM force spectroscopy (Eckel et al., 2003). This characteristic fingerprint of free dsDNA changes drastically 
(A)
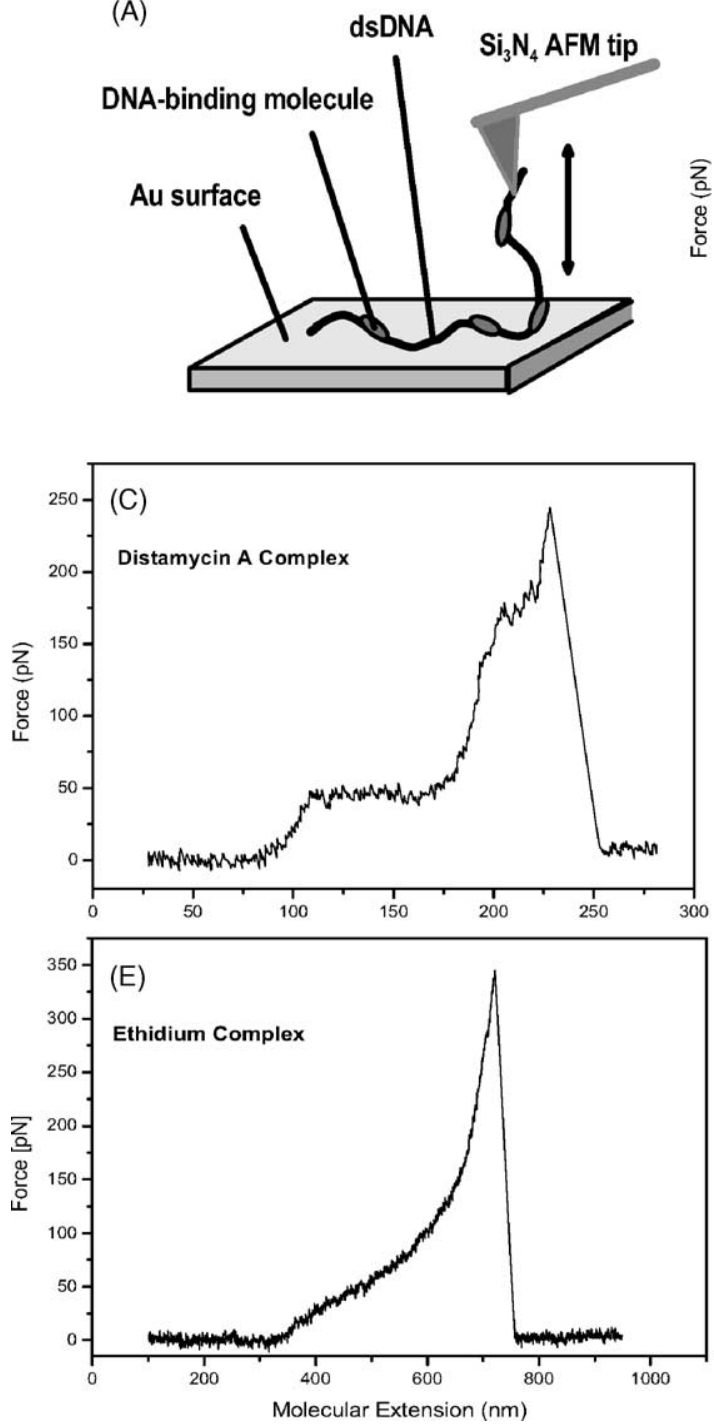
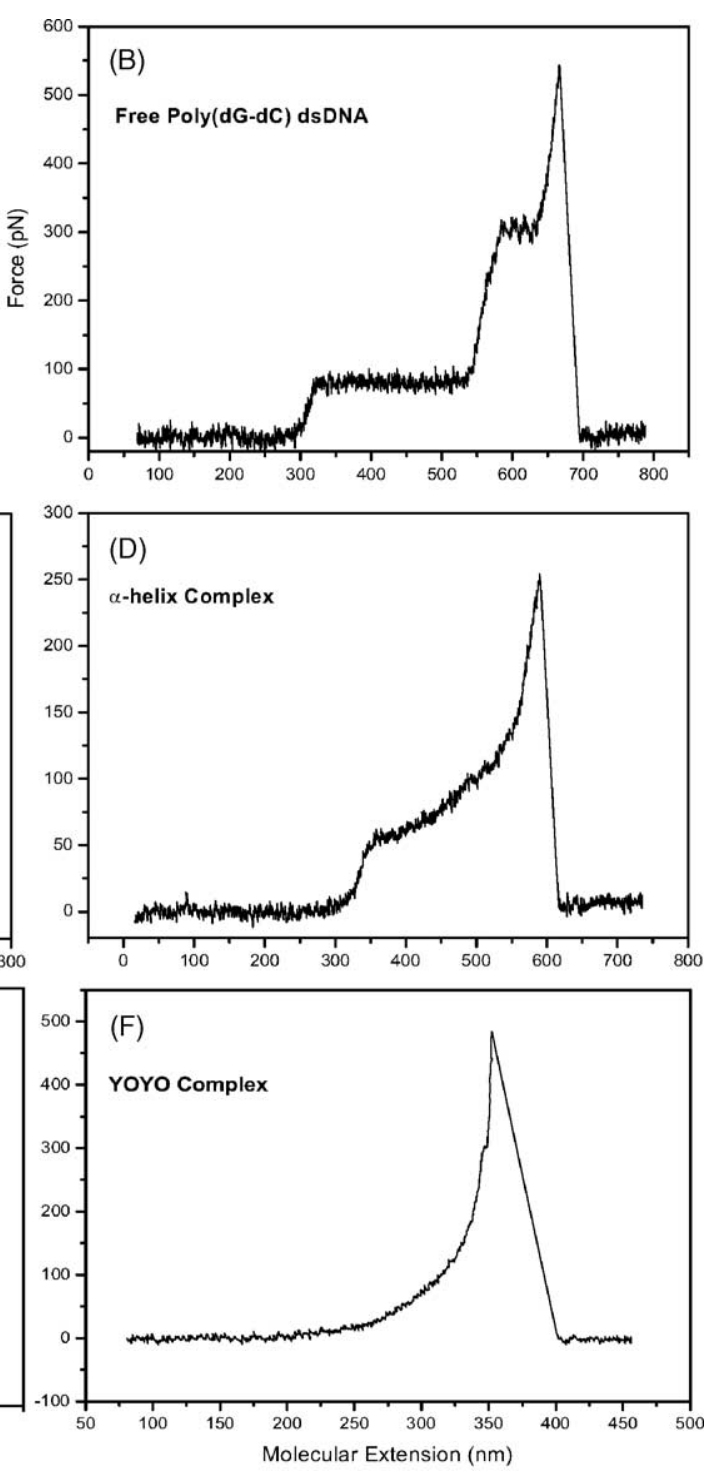

Fig. 4. Identification of binding mechanisms in single molecule-DNA complexes. (A) Experimental setup. (B) Force-extension trace of free poly(dG-dC) dsDNA. DNA complexed with (C) the minor groove binder distamycin A, (D) major groove binding $\alpha$-helical peptide, (E) with the intercalant ethidium bromide and (F) with the bis-intercalant YOYO-1. (Graphs adapted from Eckel et al., 2003.)

in the presence of small DNA binding ligands (Figs. 4 and 5). First experiments with the intercalant ethidium bromide in the low force regime were published by Cluzel et al. (Cluzel et al., 1996) and Bennink et al. with OT (Bennink et al., 1999). The force response at higher forces was studied by Anselmetti et al. (2000) and Krautbauer et al. (2000) with AFM force spectroscopy. In systematic studies Krautbauer et al. (2002a,b) investigated the crosslinking agent cisplatin, the intercalants proflavine, ethidium bromide, psoralen, and the minor groove binder netropsin, berenil and Hoechst 33258. Eckel et al. (2003) expanded the studies to major groove binding helical peptides and the bis-intercalant YOYO-1 and compared the results with the intercalants ethidium bromide, YO-1, daunomycin, and the minor groove binder distamycin 


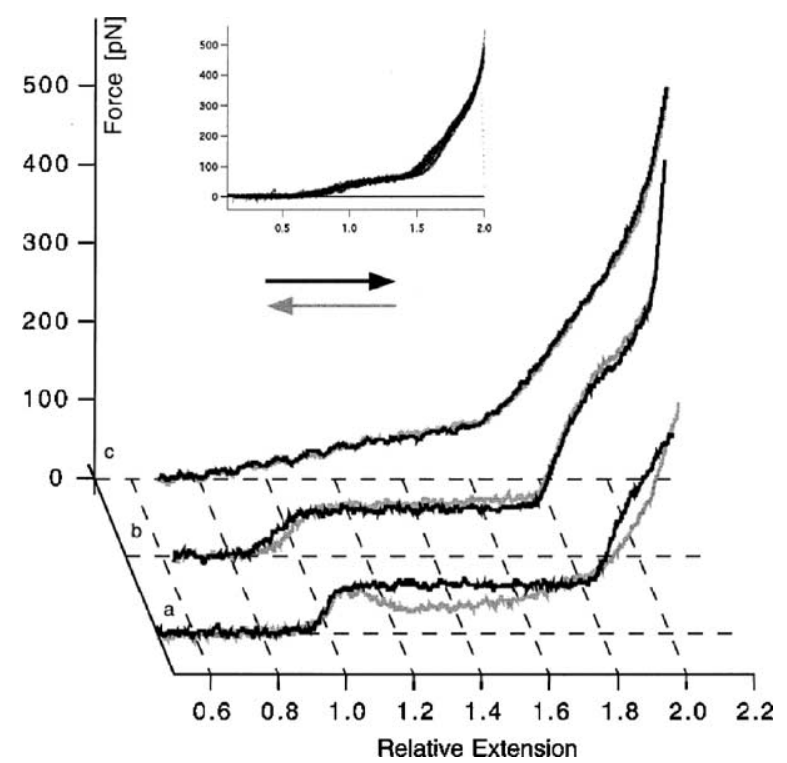

Fig. 5. Force vs. extension curves of a single $\lambda$-DNA molecule in (a) immediately after adding the crosslinking agent cisplatin (b) after $1 \mathrm{~h}$ and (c) after $24 \mathrm{~h}$. The progress of the chemical reaction is followed on the same molecule. (Graphs adapted from Krautbauer et al., 2000.)

A. In a recent work Sischka et al. (2004) investigate the influence of groove binder and intercalants with the high force resolution of an OT. The binding kinetics was addressed by time dependent elasticity measurements and the observation of hysteresis effects between stretching and relaxation processes (Husale et al., 2002; Krautbauer et al., 2002b; Sischka et al., 2004).

\subsection{Groove binding}

Binding of small, positively charged molecules may occur in the minor groove of dsDNA. This binding mode requires only slight conformational adaptions of the double helix. As for minor groove binding, major groove binding is dominated by electrostatic interactions of helical ligands with the backbone assisted by hydrogen bonds. The global characteristics of the free DNA curve are mostly conserved. The two main effects are shifting and tilting of the B-S-plateau. For the minor groove binder distamycin A with a low affinity to guanine and cytosine rich sequences, force spectroscopy with poly(dG-dC) exhibit a B-S-transition plateau without any tilt at a minimal lower force compared with free DNA (Eckel et al., 2003). In contrast, optical tweezers data for this minor groove binder with $\lambda$-DNA $(51 \% \mathrm{GC})$ indicates an increasing force value of the transition and a slight tilt that indicates a repression of the cooperative effects (Sischka et al., 2004). Similar results were found for netropsin and Hoechst 33258 (Krautbauer et al., 2002a). For the major groove binder, these two effects increase and particularly the B-S-transition is less cooperative. All groove-binding agents have no effect on the contour length of the dsDNA.

\subsection{Intercalation}

Intercalation is a different mode of interaction of small molecules with DNA. It is characterized by the sliding-in of flat, planar molecules into the base pair stack of dsDNA via interaction of their aromatic ring systems with the $\pi$-systems of the adjacent base pairs. Various intercalators have been investigated with single molecule force spectroscopy. Different groups studied ethidium bromide, a frequently used and well-characterized fluorescent staining agent for DNA, in good agreement. No distinct cooperative transition is visible and the contour length of the molecule increases dramatically. The fingerprint is clearly distinguishable from the free or groove binding curve. (Husale et al., 2002; Krautbauer et al., 2002b; Eckel et al., 2003; Sischka et al., 2004) Molecules which bind in a bis-intercalation binding mode enforce these effects of typical intercalating molecules (Eckel et al., 2003; Sischka et al., 2004).

\subsection{Crosslinking}

Crosslinking has similar effects as intercalation. The cooperative transition vanishes and the contour length increases. Krautbauer et al. (2000) investigated the prominent anti cancer drug cisplatin, which crosslinks preferentially the N7 atoms of guanine bases. Fig. 6 summarizes the mechanical fingerprints of minor- and major groove binders, inter- and bisintercalators (Sischka et al., 2004). The DNA is immobilized between two beads and the fluid chamber system allows an efficient exchange of binding agents. The force response shows distinct changes from 


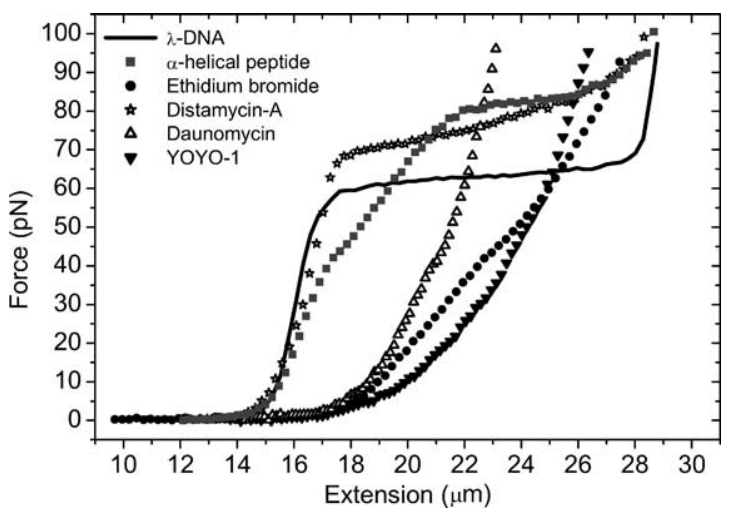

Fig. 6. Single molecule biosensor. Force response of a single $\lambda$-DNA molecule in the presence of different binding agents. The trace of the free DNA is very well distinguishable from the traces of the complexed DNA. Furthermore the force response of the minor groove binder (distamycin A), the major groove binder ( $\alpha$-helical peptide) and the intercalants or bis-intercalants (ethidium bromide, daunomycin, and YOYO-1) show characteristic fingerprints (Sischka et al., 2004).

free DNA to DNA complexes with all investigated molecules. In addition the shape of the curve allows prediction of the mode of binding and time dependent measurements provide insights into the kinetics. The combination of these features turns this setup into a promising tool for single molecule biosensing.

\section{Conclusion}

Single molecule force spectroscopy based on AFM and OT provides an ultrasensitive and powerful tool to investigate sequence specific and unspecific interactions between ligands and dsDNA. Information about kinetics, equilibrium constants, the energy landscape and the binding site can be extracted from experiments with sequence specific DNA binding proteins resulting in deeper insights for instance in the mode of transcriptional regulation. Binding modes like intercalation, groove binding and cross linking of small ligands can be explored in a very fast and efficient manner by observing changes in the molecular elasticity of the associated DNA molecule, which may lead to new biosensor applications.

\section{References}

Anselmetti, D., Fritz, J., Smith, B., Fernandez-Busquets, X., 2000. Single molecule DNA biophysics with atomic force mictroscopy. Single Mol. 1, 17-23.

Ashkin, A., 1997. Optical trapping and manipulation of neutral particles using lasers. Proc. Natl. Acad. Sci. U.S.A. 94, 48534860 .

Ashkin, A., Dziedzic, J., Bjorkholm, J., Chu, S., 1986. Observation of a single-beam gradient force optical trap for dielectric particles. Opt. Lett. 11, 288-290.

Bartels, F.W., Baumgarth, B., Anselmetti, D., Ros, R., Becker, A., 2003. Specific binding of the regulatory protein ExpG to promoter regions of the galactoglucan biosynthesis gene cluster of Sinorhizobium meliloti-a combined molecular biology and force spectroscopy investigation. J. Struct. Biol. 143, 145-152.

Bennink, M.L., Schärer, O.D., Kanaar, R., Sakata-Sogawa, K., Schins, J.M., Kanger, J.S., de Grooth, B.G., Greve, J., 1999. Single-molecule manipulation of double-stranded DNA using optical tweezers: interaction studies of DNA with RecA and YOYO-1. Cytometry 36, 200-208.

Binnig, G., Quate, C.F., Gerber, C., 1986. Atomic force microscope. Phys. Rev. Lett. 56, 930-933.

Bustamante, C., 1994. Entropic elasticity of lambda-phage DNA. Science 265, 1599-1600.

Bustamante, C., Macosko, J.C., Wuite, G.J., 2000a. Grabbing the cat by the tail: manipulating molecules one by one. Nat. Rev. Mol. Cell Biol. 1, 130-136.

Bustamante, C., Smith, S.B., Liphardt, J., Smith, D., 2000 b. Single-molecule studies of DNA mechanics. Curr. Opin. Struct. Biol. 10, 279-285.

Cluzel, P., Lebrun, A., Heller, C., Lavery, R., Viovy, J.-L., Chatenay, D., Caron, F., 1996. DNA: an extensible molecule. Science 271, 792-794.

Dammer, U., Hegner, M., Anselmetti, D., Wagner, P., Dreier, M., Huber, W., Güntherodt, H.-J., 1996. Specific antigen/antibody interactions measured by force microscopy. Biophys. J. 70, 2437-2441.

Eckel, R., Ros, R., Ros, A., Wilking, S.D., Sewald, N., Anselmetti, D., 2003. Identification of binding mechanisms in single molecule-DNA complexes. Biophys. J. 85, 1968-1973.

Evans, E., 2001. Probing the relation between force-lifetime-and chemistry in single molecular bonds. Annu. Rev. Biophys. Biomol. Struct. 30, 105-128.

Evans, E., Ritchie, K., 1997. Dynamic strength of molecular adhesion bonds. Biophys. J. 72, 1541-1555.

Evstigneev, M., Reimann, P., 2003. Dynamic force spectroscopy: optimized data analysis. Phys. Rev. E 68, 045103.

Florin, E.-L., Moy, V.T., Gaub, H.E., 1994. Adhesion forces between individual ligand-receptor pairs. Science 264, 415417.

Fritz, J., Katopodis, A.G., Kolbinger, F., Anselmetti, D., 1998. Force-mediated kinetics of single P-selectin/ligand complexes observed by atomic force microscopy. Proc. Natl. Acad. Sci. U.S.A. 95, 12283-12288.

Gordon, J.P., 1973. Radiation forces and momenta in dielectric media. Phys. Rev. A 8, 14-21. 
Hinterdorfer, P., Baumgartner, W., Gruber, H., Schilcher, K., Schindler, H., 1996. Detection and localization of individual antibody-antigen recognition events by atomic force microscopy. Proc. Natl. Acad. Sci. U.S.A. 93, 3477-3481.

Husale, S., Grange, W., Hegner, M., 2002. DNA mechanics affected by small DNA intercalating ligands. Single Mol. 3, 91-96.

Koch, S.J., Shundrovsky, A., Jantzen, B.C., Wang, M., 2002. Probing protein-DNA interactions by unzipping a single DNA double helix. Biophys. J. 83, 1098-1105.

Koch, S.J., Wang, M.D., 2003. Dynamic force spectroscopy of protein-DNA interactions by unzipping DNA. Phys. Rev. Lett. 91, 208103.

Krautbauer, R., Clausen-Schaumann, H., Gaub, H.E., 2000. Cisplatin changes the mechanics of single DNA molecules. Angew. Chem. Int. Ed. 39, 3912-3915.

Krautbauer, R., Fischerländer, S., Allen, S., Gaub, H.E., 2002a. Mechanical fingerprints of DNA drug complexes. Single Mol. 3, 97-103.

Krautbauer, R., Pope, L.H., Schrader, T.E., Allen, S., Gaub, H.E., 2002b. Discriminating small molecule DNA binding modes by single molecule force spectroscopy. FEBS Lett. 510, 154-158.

Lee, G.U., Chrisey, L.A., Colton, R.J., 1994. Direct measurement of the forces between complementary strands of DNA. Science 266, 771-773.

Lee, G.U., Kidwell, D.A., Colton, R.J., 1993. Sensing discrete streptavidin-biotin interactions with atomic force microscopy. Langmuir 10, 354-357.

Marszalek, P.E., Lu, H., Li, H., Carrion-Vazquez, M., Oberhauser, A.F., Schulten, K., Fernandez, J.M., 1999. Mechanical unfolding intermediates in titin modules. Nature 402, 100-103.

Merkel, R., Nassoy, P., Leung, A., Ritchie, K., Evans, E., 1999. Energy landscapes of receptor-ligand bonds explored with dynamic force spectroscopy. Nature 397, 50-53.
Meyer, G., Amer, N.M., 1988. Novel optical approach to atomic force microscopy. Appl. Phys. Lett. 53, 1045-1047.

Rief, M., Clausen-Schaumann, H., Gaub, H.E., 1999. Sequence-dependent mechanics of single DNA molecules. Nat. Struct. Biol. 6, 346-349.

Rief, M., Gautel, M., Oesterhelt, F., Fernandez, J.M., Gaub, H.E., 1997a. Reversible unfolding of individual titin immunoglobulin domains by AFM. Science 276, 1109-1112.

Rief, M., Oesterhelt, F., Heymann, B., Gaub, H.E., 1997b. Single molecule force spectroscopy on polysaccharides by atomic force microscopy. Science 275, 1295-1297.

Ros, R., Schwesinger, F., Anselmetti, D., Kubon, M., Schäfer, R., Plückthun, A., Tiefenauer, L., 1998. Antigen binding forces of individually addressed single-chain Fv antibody molecules. Proc. Natl. Acad. Sci. U.S.A. 95, 7402-7405.

Schwesinger, F., Ros, R., Strunz, T., Anselmetti, D., Güntherodt, H.-J., Honegger, A., Jermutus, L., Tiefenauer, L., Plückthun, A., 2000. Unbinding forces of single antibody-antigen complexes correlate with their thermal dissociation rates. Proc. Natl. Acad. Sci. U.S.A. 97, 9972-9977.

Sischka, A., Tönsing, K., Eckel, R., Wilking, S.D., Sewald, N., Ros, R., Anselmetti, D., 2004. Molecular mechanisms and kinetics between DNA and DNA binding ligands, submitted.

Smith, S.B., Cui, Y., Bustamante, C., 1996. Overstretching B-DNA: the elastic response of individual double-stranded and single-stranded DNA molecules. Science 271, 795799.

Strunz, T., Oroszlan, K., Schäfer, R., Güntherodt, H.-J., 1999. Dynamic force spectroscopy of single DNA molecules. Proc. Natl. Acad. Sci. U.S.A. 96, 11277-11282.

Wenner, J.R., Williams, M.C., Rouzina, J., Bloomfield, V.A., 2002. Salt dependence of the elasticity and overstretching transition of single DNA molecules. Biophys. J. 82, 3160-3169. 